\title{
PLANTAS ARVENSES ASOCIADAS A CULTIVOS DE MAÍZ DE TEMPORAL EN SUELOS SALINOS DE LA RIBERA DEL LAGO DE CUITZEO, MICHOACÁN, MÉXICO
}

\author{
Judith Sánchez-Blanco ${ }^{1,2}$ y Fernando Guevara-FÉfer ${ }^{1}$ \\ ${ }^{1}$ Universidad Michoacana de San Nicolás de Hidalgo, Facultad de Biología, \\ Laboratorio de Sinecología, Morelia, Michoacán, México. \\ ²Autor para la correspondencia: blancojudith@yahoo.com.mx
}

\section{RESUMEN}

Se muestrearon comunidades de plantas arvenses en cultivos de maíz de temporal creciendo sobre suelos con problemas de salinidad, durante el ciclo agrícola 1996 en 10 sitios de la ribera del Lago de Cuitzeo. Se registraron 133 especies correspondientes a 94 géneros y 34 familias. De estas últimas las que presentaron el mayor número de especies fueron Compositae, Gramineae, Euphorbiaceae, Solanaceae y Leguminosae que en conjunto representan más de la mitad de la riqueza específica con $63.89 \%$. Del total de plantas, $74.43 \%$ son dicotiledóneas y $25.56 \%$ monocotiledóneas. La diversidad es menor en nuestra área de estudio en comparación con el promedio de esta medida encontrado en otras áreas no salinas de la Cuenca de Cuitzeo. De las especies, 17.3\% (23) son introducidas, dominando las nativas con $82.7 \%$ (110) de América, principalmente México y áreas adyacentes. La forma de vida prevaleciente es la anual, representada por $60.90 \%$ de las plantas, seguida de la perenne $26.32 \%$, la anual-perenne $10.53 \%$ y anual-bianual $2.25 \%$. La similitud florística fue poco notable entre localidades, pues varió de 0.507 a 0.083 con el Índice de Sorensen. No se encontró correlación entre la composición y riqueza de especies y los factores fisicoquímicos del suelo; sin embargo, algunas presentaron preferencias de $\mathrm{pH}$ y textura.

Palabras clave: composición florística, malezas, parcelas agrícolas.

\section{ABSTRACT}

We sampled the weed communities of rain-fed maize growing in soils with salinity problems during the 1996 agricultural cycle in ten sites at the Lake Cuitzeo shore. We 
found 133 species belonging to 94 genera and 34 families. The families with most species were Compositae, Gramineae, Euphorbiaceae, Solanaceae and Leguminosae, together representing more than half of the specific diversity, $63.89 \%$. Dicotyledons represent $74.43 \%$ of the species, while $25.56 \%$ are Monocotyledons. Weed diversity in our study sites appear to be lower than the average diversity found in non saline sites in other areas of the Cuitzeo basin. $17.3 \%$ (23) of the species are introduced; while the remainder are plants native to the Americas $82.7 \%$ (110), principally Mexico and adjacent areas. The predominant life form is annual with $60.90 \%$, followed by the perennial forms with $26.32 \%$; the rest are facultative like annual-perennials $10.53 \%$ and annual-biannuals $2.25 \%$. The floristic similarity among the sites using the Index of Sorensen varied from 0.507 to 0.083 . No composition and diversity species correlation was found with physical-chemical factors of soils; however, some species showed preference to specific soil $\mathrm{pH}$ and texture.

Key words: floristic composition, maize plots, weeds.

\section{INTRODUCCIÓN}

En las últimas décadas, el Lago de Cuitzeo ha disminuido drásticamente su profundidad y superficie hasta en $70 \%$, afectando a 5000 familias de pescadores (Chávez, 1996a). El descenso en el nivel del agua en la época de sequía, aunado al fenómeno de hipertroficación, ha dejado al descubierto grandes superficies que conforman la ribera, impregnadas de sales poco favorables para la agricultura. Además, algunas áreas adyacentes al lago, dependiendo del nivel del agua en determinada época, son inundadas, por lo que la salinidad disminuye su fertilidad y el potencial productivo. Sin embargo, los lugareños se han visto en la necesidad de rehabilitarlas y aprovecharlas para la agricultura. La presencia de algunas plantas arvenses constituye un factor que limita el desarrollo del cultivo de maíz, ya que compiten por agua, luz y nutrientes. No obstante y a pesar de que su presencia en los cultivos puede ser nociva, cumplen funciones ecológicas importantes: son pioneras y colonizadoras en procesos de sucesión en áreas perturbadas, con sus sistemas radiculares retienen el suelo evitando la erosión, sirven de alimento a fitófagos y plagas de cultivos, proveen de néctar y polen a insectos cosechadores de miel, y fijan nitrógeno e incorporan materia orgánica ayudando a la formación del suelo (Espinosa-García, 1981; Sen, 1981).

Este grupo heterogéneo de plantas en la agricultura tradicional es parte importante de los agroecosistemas; constituyen recursos alternativos, ya que son em- 
pleadas como ornato, medicamento y alimento para el hombre, forraje para el ganado y fuente de nuevos cultivares (Alanís, 1974; Espinosa-García y Díaz, 1996; VieyraOdilon y Vibrans, 2001). Algunas desempeñan un papel importante al influir en el crecimiento de otras malezas y/o cultivos a través de la liberación de compuestos alelopáticos, y varias permiten el desarrollo de insectos benéficos que regulan las poblaciones de aquellos que son plagas (Altieri, 1987; Torres, 1991).

El presente estudio tiene la finalidad de contribuir al conocimiento florístico y ecológico de las especies arvenses en cultivos de maíz de temporal en suelos salinos de la ribera del lago, que permita llevar a cabo estudios posteriores orientados a hacer un adecuado manejo y control de las mismas.

\section{Descripción del área de estudio}

El Lago de Cuitzeo se localiza en la parte noreste del estado de Michoacán y al sur de la Altiplanicie Mexicana, se ubica a $1820 \mathrm{~m}$ sobre el nivel del mar y a 20 $\mathrm{km}$ al norte de la capital del estado (Fig. 1). Es cruzado por las carreteras federal No. 43 y la autopista Morelia-Salamanca que comunican al norte de Michoacán con el sur del estado de Guanajuato, dividiendo al lago en dos vasos, este y oeste, unidos por un ducto que permite el paso del agua que fluye en dirección este-oeste. Se ubica geográficamente entre los paralelos $19^{\circ} 53^{\prime} 15^{\prime \prime}$ y $20^{\circ} 04^{\prime} 34^{\prime \prime}$ de latitud Norte y entre los meridianos 10050'20" y 101'19'34" de longitud Oeste del Meridiano de Greenwich (Alvarado et al., 1984). Forma parte de los municipios de: Copándaro de Galeana, Cuitzeo, Chucándiro, Huandacareo, Queréndaro, Santa Ana Maya, Zinapécuaro y Álvaro Obregón en Michoacán, y el de Acámbaro en el estado de Guanajuato. Tiene una superficie aproximada de $1146 \mathrm{~km}^{2}$. La cuenca del Lago de Cuitzeo se ubica en la provincia fisiográfica del Eje Volcánico Transversal y en la Cuenca Lerma-Chapala-Santiago. Originalmente era una cuenca cerrada o endorreica; debido a los fenómenos tectónicos y volcánicos, fue abierta artificialmente durante la época de la colonia, dándole una salida hacia el río Lerma por medio del canal La Cinta al norte. Tiene como principales afluentes por el sur el río Grande de Morelia y por el sureste, los ríos Zinapécuaro y Queréndaro (Chávez, 1996b).

En los alrededores del Lago de Cuitzeo predominan suelos de tipo vertisol pélico, con textura fina, abundante arcilla y gran cantidad de sal (cloruro de sodio), tequesquite (mezcla de carbonato y bicarbonato de sodio) y salmuera (mezcla de sulfato y cloruro de sodio), que impregnan los terrenos adyacentes al vaso (Corona, 1976). También se encuentran feozem háplico, feozem lúvico, litosol, vertisol pélico-fase sódica, gleysol mólico y solonchak gléyico-fase sódica con textura fina. 


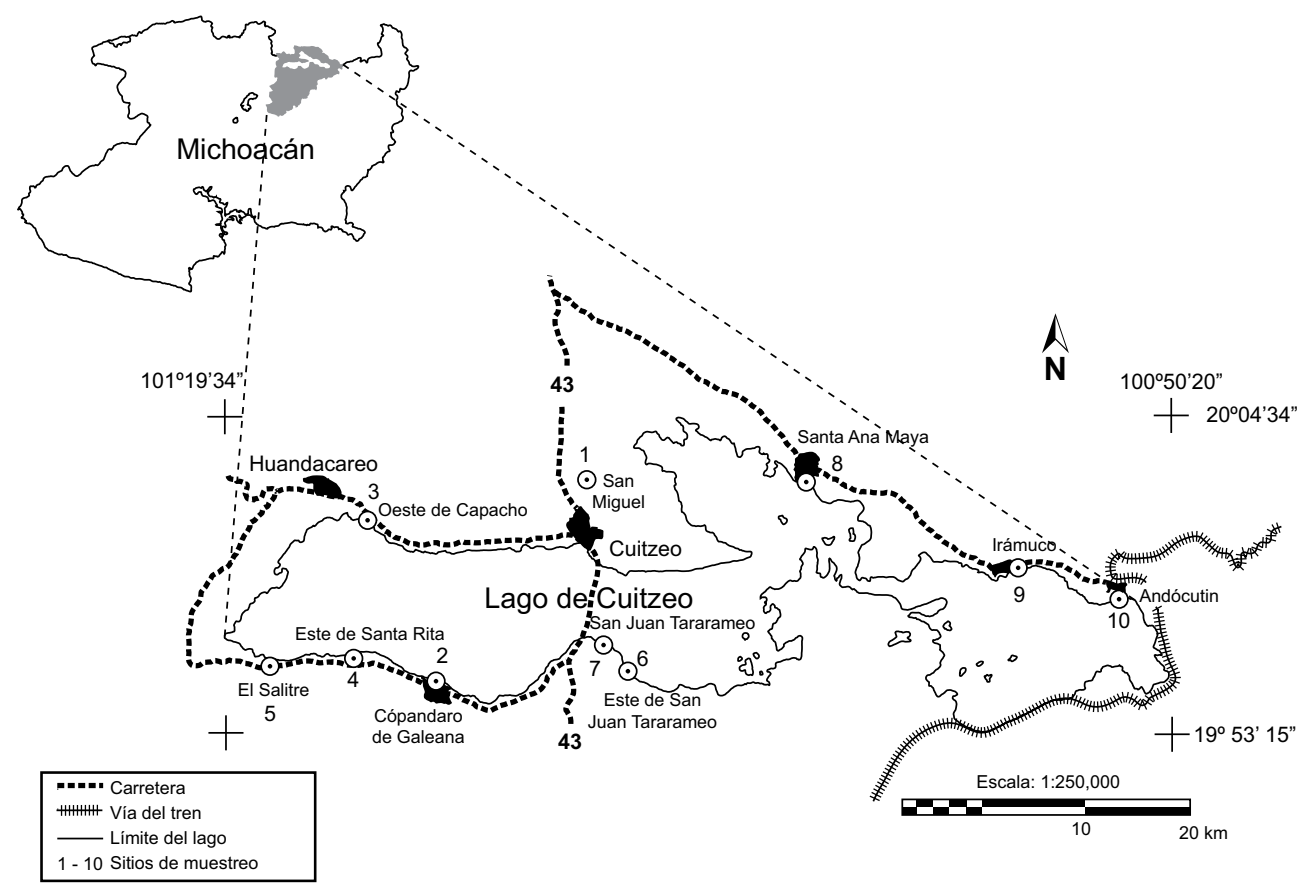

Fig. 1. Ubicación de las parcelas muestreadas.

El clima dominante en la región corresponde al $\mathrm{Cw}_{\mathrm{o}}(\mathrm{w}) \mathrm{b}\left(\mathrm{i}^{\prime}\right) \mathrm{g}$, templado subhúmedo con lluvias en verano. Los datos climatológicos existentes corresponden a cuatro estaciones distribuidas en el área de estudio (García, 1988), los cuales se resumen en el Cuadro 1 ; se aprecia que la temperatura media anual varía de $16.8^{\circ} \mathrm{C}$ (Copándaro) a $18.6^{\circ} \mathrm{C}$ (Irámuco).

La precipitación pluvial en promedio anual registra valores de $669.8 \mathrm{~mm}$ (Cuitzeo) a 959 mm (Chucándiro), el mes más lluvioso es julio y el más seco marzo. El sector norte y oriente es el más seco, y el sur y poniente el más húmedo.

En las inmediaciones del lago se reconocen tres tipos de vegetación: pastizal halófilo, bosque espinoso, bosque tropical caducifolio, este último con cuatro facetas (variante típica, así como tres etapas secundarias, a mencionar matorral subtropical, matorral espinoso y pastizal inducido). El bosque tropical caducifolio y sus fases sucesionales ocupan la mayor superficie en los lomeríos que rodean la zona de estudio (Guevara-Féfer, 1995). 
Cuadro 1. Resumen de los datos climáticos de las estaciones ubicadas en la ribera del Lago de Cuitzeo (García, 1988).

\begin{tabular}{lccrl}
\hline Estación climática & Altitud $(\mathrm{m})$ & $\begin{array}{c}\text { Temperatura } \\
\text { promedio anual }\end{array}$ & $\begin{array}{c}\text { Precipitación } \\
\text { anual }\end{array}$ & Subtipo \\
\hline Copándaro & 1826 & $16.8^{\circ} \mathrm{C}$ & $788.1 \mathrm{~mm}$ & $\mathrm{Cb}\left(\mathrm{w}_{1}\right) \mathrm{w}\left(\mathrm{i}^{\prime}\right) \mathrm{g}$ \\
Cuitzeo & 1831 & $17.9^{\circ} \mathrm{C}$ & $669.8 \mathrm{~mm}$ & $\mathrm{Cb}\left(\mathrm{w}_{\mathrm{o}}\right)(\mathrm{w})(\mathrm{e}) \mathrm{g}$ \\
Chucándiro & 1840 & $17.4^{\circ} \mathrm{C}$ & $959 \mathrm{~mm}$ & $\mathrm{Cb}\left(\mathrm{w}_{1}\right)(\mathrm{w})(\mathrm{e}) \mathrm{g}$ \\
Irámuco & 1870 & $18.6^{\circ} \mathrm{C}$ & $725 \mathrm{~mm}$ & $(\mathrm{~A}) \mathrm{Cb}\left(\mathrm{w}_{\mathrm{o}}\right)(\mathrm{w})\left(\mathrm{i}^{\prime}\right) \mathrm{g}$ \\
\hline
\end{tabular}

\section{MÉTODOS}

Se hicieron recorridos de reconocimiento del área para ubicar las parcelas agrícolas de muestreo, de acuerdo con su accesibilidad. Se ubicaron 10 sitios de inventario (Cuadro 2), tratando de abarcar las áreas de la ribera este y oeste del lago con problemas de salinidad (Fig. 1).

Los muestreos se efectuaron durante los meses de octubre y noviembre de 1996 en diferentes parcelas de maíz de temporal, que correspondieron al cierre del ciclo agrícola, cuando las comunidades de plantas arvenses han alcanzado la madurez.

Se utilizó el método de cuadros al azar, que consiste en colocar aleatoriamente cuadros (en nuestro caso círculos) repetidamente en la comunidad (Mueller-Dombois y Ellenberg, 1974). El área de dichos círculos fue de $0.25 \mathrm{~m}^{2} \mathrm{c} / \mathrm{u}$, considerándola como el tamaño adecuado para muestrear este tipo de vegetación, de acuerdo con los trabajos previos en la cuenca (Guevara-Féfer et al., 1984; Chávez, 1996b).

Los censos en cada milpa se iniciaron a partir de un metro de la orilla hacia el centro del cultivo para evitar el efecto de orilla. Se inventariaron 10 círculos por parcela de maíz, cubriendo una superficie total de $2.5 \mathrm{~m}^{2}$ por solar. Todas las plantas arvenses que se encontraron se colectaron y colocaron en una bolsa de plástico debidamente etiquetada. Después se hicieron recorridos en la milpa para recolectar ejemplares de las malezas que no se habían encontrado dentro de los cuadros muestreados. Cuando fue posible se entrevistó al dueño del terreno y a los lugareños, para obtener información sobre labores culturales, el nombre común de las especies, su uso y otros datos. En el laboratorio el material colectado por sitio se separó; su identificación se hizo usando los trabajos de Rzedowski y Rzedows- 
Cuadro 2. Sitios de muestreo, pH y tipos de suelo en cultivos de maíz de temporal de la ribera del Lago de Cuitzeo, Michoacán.

\begin{tabular}{|c|c|c|c|}
\hline Sitio & Textura & $\mathrm{pH}$ & Tipo de suelo \\
\hline 1. San Miguel & Franco arenoso & 8.7 & $\begin{array}{l}\text { Vertisol pélico+feozem salino } \\
\text { y sódico }\end{array}$ \\
\hline $\begin{array}{l}\text { 2. Copándaro de } \\
\text { Galeana }\end{array}$ & Franco & 8.7 & Vertisol pélico \\
\hline 3. Oeste de Capacho & $\begin{array}{l}\text { Franco arcilloso- } \\
\text { arenoso }\end{array}$ & 6.7 & Vertisol pélico+litosol \\
\hline 4. Este de Santa Rita & Franco arcilloso & 9.1 & Vertisol pélico+feozem háplico \\
\hline 5. El Salitre & Arcilloso & 7.3 & Vertisol pélico+feozem háplico \\
\hline $\begin{array}{l}\text { 6. Este de San Juan } \\
\text { Tararameo }\end{array}$ & Franco & 9.0 & Vertisol pélico+feozem háplico \\
\hline 7. San Juan Tararameo & Arcilloso & 9.0 & Vertisol pélico+feozem háplico \\
\hline 8. Santa Ana Maya & Arcilloso & 8.0 & Vertisol pélico \\
\hline 9. Irámuco & Franco arcilloso & 8.7 & Vertisol pélico+feozem lúvico \\
\hline 10. Andócutin & Franco arcilloso & 7.4 & Vertisol pélico \\
\hline
\end{tabular}

ki (1979, 1985 y 1990), McVaugh (1983 y 1984), Beetle et al. (1983, 1987, 1991 y 1995) y Espinosa-García y Sarukhán (1997) y se cotejó con la revisión de especímenes en los herbarios del Centro Regional del Bajío, del Instituto de Ecología, A.C. (IEB) y de la Facultad de Biología, Universidad Michoacana de San Nicolás de Hidalgo (EBUM), donde se depositaron.

También se tomaron muestras al azar de la capa arable del suelo dentro de cada milpa para el estudio físico-químico del mismo, con un cilindro metálico de $7.8 \mathrm{~cm}$ de diámetro y $15 \mathrm{~cm}$ de largo, con capacidad de $800 \mathrm{~g}$. Éstas se analizaron en el laboratorio de suelos del Distrito de Desarrollo Rural Morelia-Queréndaro No. 092.

Para definir la similitud florística se utilizó el Índice modificado de Sorensen, basado solamente en datos de ausencia-presencia de las especies en las comunidades (Krebs, 1989). El cálculo y la elaboración del respectivo dendrograma se realizó con el programa MVSP versión 3.1 (Kovach, 2005) mediante la técnica de agrupamiento por ligamiento promedio no ponderado, también conocido como método de análisis por grupos pareados no ponderado (UPGMA). 
Sánchez-Blanco y Guevara-Féfer: Plantas arvenses asociadas a cultivos de maíz en suelos salinos

$$
I S=\frac{2 a}{2 a+b+c}
$$

$\mathrm{a}=$ número de especies que existen en las muestras $a$ y $b$

$\mathrm{b}=$ número de especies presentes en la muestra $b$ pero no en la $a$

$\mathrm{c}=$ número de especies revisadas en la muestra $a$ pero no en la $b$

\section{RESULTADOS Y DISCUSIÓN}

Se registraron 133 especies incluyendo 2 subespecies, agrupadas en 94 géneros y 34 familias de fanerógamas (Apéndice). De éstas, 23 son introducidas conformando $17.3 \%$ del total, las nativas dominan con $82.7 \%$ (110 especies).

Las familias con mayor número de especies son Compositae y Gramineae con 29 cada una (21.80\%), Euphorbiaceae con 14 (10.52\%), Solanaceae con $7(5.26 \%)$ y Leguminosae con 6 (4.51\%), que en conjunto constituyen $63.89 \%$ del total registrado; con cuatro especies se encontraron a Polygonaceae y Malvaceae, con tres Onagraceae y Oxalidaceae, con dos Scrophulariaceae, Amaranthaceae, Commelinaceae, Convolvulaceae, Cyperaceae, Labiatae, Loasaceae, Umbelliferae, Verbenaceae; las demás familias están representadas por una sola (Cuadro 3).

Lo encontrado en el área de estudio y en otras dos zonas cercanas (Chávez y Guevara-Féfer, 2003; Bernal y Guevara-Féfer, 2003) ubica a Compositae y Gramineae como las más diversas, que junto con Euphorbiaceae, Solanaceae y Leguminosae, constituyen los grupos dominantes en los tres lugares. El género con mayor número de taxa fue Euphorbia con 9 especies, seguido por Acalypha y Melampodium con 4, y Oxalis, Chloris, Panicum, Paspalum y Setaria con 3. Estos números son similares a los obtenidos para el Valle de Morelia (Chávez y Guevara-Féfer, 2003) y mayores que los consignados para Villa Morelos (Bernal y Guevara-Féfer, 2003). Las semejanzas con el primero pueden ser atribuibles a que ambas áreas de estudio se encuentran a altitudes similares, mientras que las diferencias con la segunda se deben probablemente a que esta área se encuentra cercana a los $2500 \mathrm{~m}$ sobre el nivel del mar y el clima es más húmedo.

Del total de las especies $74.43 \%$ lo conforman las dicotiledóneas y el $25.56 \%$ restante las monocotiledóneas (Cuadro 4). Dichos valores son parecidos a los reportados para el Valle de Morelia y Villa Morelos en el estado de Michoacán (Chávez y Guevara-Féfer, 2003 y Bernal y Guevara-Féfer, 2003). 
Cuadro 3. Participación cuantitativa de las familias de la flora estudiada, con base en el número de géneros y de especies.

\begin{tabular}{|c|c|c|c|c|}
\hline Familia & Géneros & $\%$ & Especies & $\%$ \\
\hline Compositae & 22 & 23.40 & 29 & 21.80 \\
\hline Gramineae & 18 & 19.15 & 29 & 21.80 \\
\hline Euphorbiaceae & 3 & 3.19 & 14 & 10.52 \\
\hline Solanaceae & 5 & 5.30 & 7 & 5.26 \\
\hline Leguminosae & 5 & 5.30 & 6 & 4.51 \\
\hline Malvaceae & 3 & 3.19 & 4 & 3.00 \\
\hline Polygonaceae & 2 & 2.13 & 4 & 3.00 \\
\hline Onagraceae & 2 & 2.13 & 3 & 2.25 \\
\hline Amaranthaceae & 2 & 2.13 & 2 & 1.50 \\
\hline Commelinaceae & 2 & 2.13 & 2 & 1.50 \\
\hline Cyperaceae & 2 & 2.13 & 2 & 1.50 \\
\hline Labiatae & 2 & 2.13 & 2 & 1.50 \\
\hline Loasaceae & 2 & 2.13 & 2 & 1.50 \\
\hline Scrophulariaceae & 2 & 2.13 & 2 & 1.50 \\
\hline Umbelliferae & 2 & 2.13 & 2 & 1.50 \\
\hline Verbenaceae & 2 & 2.13 & 2 & 1.50 \\
\hline Oxalidaceae & 1 & 1.06 & 3 & 2.25 \\
\hline Convolvulaceae & 1 & 1.06 & 2 & 1.50 \\
\hline Acanthaceae & 1 & 1.06 & 1 & 0.75 \\
\hline Aizoaceae & 1 & 1.06 & 1 & 0.75 \\
\hline Boraginaceae & 1 & 1.06 & 1 & 0.75 \\
\hline Chenopodiaceae & 1 & 1.06 & 1 & 0.75 \\
\hline Cruciferae & 1 & 1.06 & 1 & 0.75 \\
\hline Cucurbitaceae & 1 & 1.06 & 1 & 0.75 \\
\hline Nyctaginaceae & 1 & 1.06 & 1 & 0.75 \\
\hline Papaveraceae & 1 & 1.06 & 1 & 0.75 \\
\hline Passifloraceae & 1 & 1.06 & 1 & 0.75 \\
\hline Pontederiaceae & 1 & 1.06 & 1 & 0.75 \\
\hline Portulacaceae & 1 & 1.06 & 1 & 0.75 \\
\hline Plantaginaceae & 1 & 1.06 & 1 & 0.75 \\
\hline Plumbaginaceae & 1 & 1.06 & 1 & 0.75 \\
\hline Primulaceae & 1 & 1.06 & 1 & 0.75 \\
\hline Rubiaceae & 1 & 1.06 & 1 & 0.75 \\
\hline Zygophyllaceae & 1 & 1.06 & 1 & 0.75 \\
\hline TOTAL 34 & 94 & 100 & 133 & 100 \\
\hline
\end{tabular}


Cuadro 4. Participación de grandes grupos taxonómicos en la flora arvense estudiada.

\begin{tabular}{llll}
\hline Clase & Familias & \multicolumn{1}{c}{ Géneros } & Especies \\
\hline Monocotiledóneas & $3(8.82 \%)$ & $21(22.34 \%)$ & $34(25.56 \%)$ \\
Dicotiledóneas & $31(91.18 \%)$ & $73(77.66 \%)$ & $99(74.43 \%)$ \\
TOTAL & $34(100 \%)$ & $94(100 \%)$ & $133(100 \%)$ \\
\hline
\end{tabular}

En cuanto a su forma de vida, $60.90 \%$ de las plantas son anuales, $26.32 \%$ perennes, $10.53 \%$ anuales-perennes y $2.25 \%$ anuales-bianuales. Estas cantidades son muy similares a las registradas para las dos áreas anteriormente comparadas.

El número de especies por sitio es muy variable (16 a 36), siendo mayor en la localidad Este de San Juan Tararameo (36), seguido de Santa Ana Maya (27), y la menor cantidad en El Salitre y San Juan Tararameo (16). La composición florística de las diferentes comunidades analizadas en el presente estudio no es homogénea, varía de un sitio a otro.

En relación con los valores de riqueza de especies reportados en áreas aledañas que se encuentran en suelos no salinos de la Cuenca de Cuitzeo, Chávez (1996b) encontró en su estudio del Valle de Morelia que los sitios más diversos están localizados en suelos de tipo andosol con un promedio de 40 especies, seguidos de luvisol con un promedio de 33, los de tipo acrisol con 30, y los de tipo vertisol y feozem presentan valores entre 23 y 27 especies respectivamente. Bernal y Guevara-Féfer (2003) registraron para 10 parcelas en suelos de tipo vertisol un promedio de 27.5 especies por parcela. La diversidad de plantas arvenses que crecen en los suelos salinos encontradas en el presente trabajo es menor que lo registrado en los trabajos arriba mencionados (promedio de 22 especies por sitio), lo cual sugiere que tales condiciones edáficas pueden limitar la riqueza de malezas asociadas al cultivo de maíz de temporal.

Similitud florística entre las localidades

De acuerdo con la matriz de los coeficientes de similitud de Sorensen, entre las localidades muestreadas (Cuadro 5), la mayoría de los valores son menores de 0.5 , indicando una composición florística poco semejante entre los sitios estudiados, siendo el número más alto 0.507, que corresponde a Santa Ana Maya e Irámuco que tienen $\mathrm{pH}$ del suelo de 8.0 y 8.7 respectivamente, mientras que San Juan Tararameo y Andócutin presentan la similitud más baja 0.083 ; sus valores de $\mathrm{pH}$ del suelo son de 9.0 y 7.4 respectivamente. 
Cuadro 5. Matriz de los coeficientes de similitud de Sorensen entre las diferentes localidades de muestreo. Las similitudes mínima y máxima están indicadas en gris.

\begin{tabular}{lcccccccccc}
\hline Localidades & 1 & 2 & 3 & 4 & 5 & 6 & 7 & 8 & 9 & 10 \\
\hline 1 (pH 8.7) & 1 & & & & & & & & & \\
$2(\mathrm{pH} \mathrm{8.7)}$ & 0.379 & 1 & & & & & & & & \\
$3(\mathrm{pH} \mathrm{6.7)}$ & 0.348 & 0.364 & 1 & & & & & & & \\
$4(\mathrm{pH} \mathrm{9.1)}$ & 0.267 & 0.300 & 0.235 & 1 & & & & & & \\
$5(\mathrm{pH} \mathrm{7.3)}$ & 0.211 & 0.207 & 0.304 & 0.333 & 1 & & & & & \\
$6(\mathrm{pH} \mathrm{9.0)}$ & 0.389 & 0.413 & 0.425 & 0.404 & 0.278 & 1 & & & & \\
7 (pH 9.0) & 0.316 & 0.241 & 0.304 & 0.233 & 0.263 & 0.361 & 1 & & & \\
$8(\mathrm{pH} \mathrm{8.0)}$ & 0.333 & 0.375 & 0.294 & 0.341 & 0.400 & 0.340 & 0.200 & 1 & & \\
$9(\mathrm{pH} \mathrm{8.7)}$ & 0.340 & 0.356 & 0.295 & 0.427 & 0.340 & 0.46 & 0.264 & 0.507 & 1 & \\
$10(\mathrm{pH} \mathrm{7.4})$ & 0.250 & 0.265 & 0.286 & 0.257 & 0.083 & 0.317 & 0.292 & 0.257 & 0.413 & 1 \\
\hline
\end{tabular}

En el dendrograma (Fig. 2) se muestran las agrupaciones resultantes del análisis multivariado (UPGMA); se puede reconocer un primer conjunto formado por los sitios 7 y 10, el segundo está constituido por el resto de los sitios y dentro de éste se pueden reconocer dos subgrupos, uno que incluye los sitios 5, 9, 8 y 4, y el segundo $\operatorname{los}$ sitios $6,3,2$ y 1 .

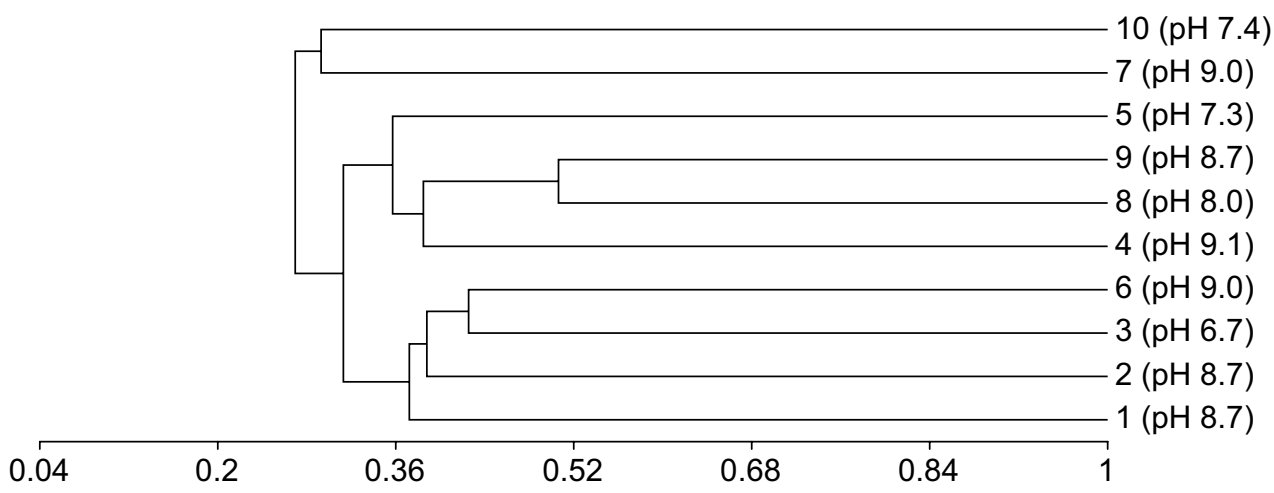

Fig. 2. Dendrograma que muestra la agrupación de localidades con base en su similitud florística. Se indican las localidades y su correspondiente valor de $\mathrm{pH}$. $(1=$ San Miguel, $2=$ Cópandaro de Galeana, 3 = Oeste de Capacho, 4 = Este de Santa Rita; 5 = El Salitre, 6 = Este de San Juan Tararameo, $7=$ San Juan Tararameo, $8=$ Santa Ana Maya, $9=$ Irámuco, $10=$ Andócutin). 
También se hicieron comparaciones con otras áreas que, al igual que la del presente estudio, forman parte de la Cuenca de Cuitzeo y en las cuales se utilizó la misma metodología de muestreo. El mayor valor de similitud de especies fue con el Valle de Morelia (0.398), y le sigue Villa Morelos (0.306); estas divergencias se deben a diferentes características ambientales propias de cada región, especialmente el suelo y clima, así como a distintas labores de manejo y control en cada área (Cuadro 6).

Relaciones y orígenes de la flora arvense

Con respecto a las relaciones y orígenes de la flora arvense de la ribera del Lago de Cuitzeo, se pueden reconocer dos grupos. El primero está constituido por plantas introducidas, provenientes de áreas tan lejanas como Europa, África, Asia, Sudamérica y otras (Villaseñor y Espinosa-García, 2004 y Rzedowski, 1991). Tal conjunto suma 23 especies, es decir 17.3\% del total, siendo la principal fuente Eurasia. Este relativamente bajo porcentaje coincide con lo estimado por Rzedowski (1991) y Chávez y Guevara-Féfer (2003), quienes encuentran que el elemento autóctono de la flora arvense es dominante sobre el introducido.

El segundo grupo está conformado por las plantas nativas que suman 110 especies (82.7\%), las cuales se distribuyen en diferentes sectores del continente americano y en climas predominantemente tropicales. De éstas, al menos la cuarta parte tiene un área restringida a México, o bien, a México y los estados limítrofes de Estados Unidos y Guatemala (Megaméxico 1 y Megaméxico 2, según Rzedowski, 1991). Estas últimas constituyen el elemento autóctono del país y representan cerca de la tercera parte del total de especies encontradas.

Del listado que proporcionan Rojas y Novelo (1995), de la flora y vegetación acuática del Lago de Cuitzeo; 18 especies se encontraron en el presente estu-

Cuadro 6. Similitud entre las floras arvenses de áreas vecinas y de la ribera del Lago de Cuitzeo, Michoacán.

\begin{tabular}{llccc}
\hline Área & Autores & $\begin{array}{c}\text { Núm. de } \\
\text { especies }\end{array}$ & $\begin{array}{c}\text { Especies } \\
\text { compartidas }\end{array}$ & $\begin{array}{c}\text { Índice de } \\
\text { similitud }\end{array}$ \\
\hline $\begin{array}{c}\text { Valle de Morelia, } \\
\text { Michoacán }\end{array}$ & Chávez, 1996 & 189 & 64 & 0.398 \\
$\begin{array}{c}\text { Villa Morelos, } \\
\text { Michoacán }\end{array}$ & $\begin{array}{c}\text { Bernal y Guevara- } \\
\text { Féfer, 2003 }\end{array}$ & 98 & 34 & 0.306 \\
\hline
\end{tabular}


dio. La mayor parte son tolerantes al medio acuoso (bajo condiciones ecológicas de alta salinidad y perturbación, cubriendo grandes áreas en el borde del lago) como Amaranthus hybridus, Heliotropium curassavicum, Chenopodium murale, Tripogandra purpurascens, Flaveria trinervia, Sanvitalia procumbens, Chloris virgata, Oenothera pubescens, Plantago major y Solanum americanum.

En la categoría de subacuáticas a tolerantes se encuentran Echinochloa colonum y Echinochloa crus-galli, las cuales presentan valores de abundancia altos dentro de los cultivos. Como subacuáticas enraizadas emergentes tenemos a Eclipta prostrata, Bacopa monnieri e Hydrocotyle ranunculoides (a veces de hojas flotantes), así como a Schoenoplectus americanus y Leersia hexandra; como acuática libremente flotadora se registra a Eichhornia crassipes.

Rojas y Novelo (1995) reportan también que las especies de esta última categoría cubren grandes áreas con altos contenidos de sales en el borde del lago durante períodos de sequía. Tal hecho favorece su colonización y establecimiento en los cultivos.

\section{Nuevos registros}

En el Apéndice, las especies marcadas con asterisco y el signo de más suman 32 y se consideran nuevos registros como plantas arvenses de cultivos de maíz, de acuerdo con los listados consultados para el país (Villaseñor y EspinosaGarcía, 1998); de la cuenca (Chávez y Guevara-Féfer, 2003) y otras regiones del mundo (Holm et al., 1979; Marzocca, 1976); de éstas habría que excluir a aquellas (principalmente) leñosas y herbáceas perennes que más bien son colonizadoras de campos abandonados o de vestigios de vegetación natural (+). Excluyendo las últimas, quedan 27 como posibles nuevos registros de plantas arvenses en cultivos de maíz de temporal para México. Estas especies presentan valores de abundancia muy bajos, por lo que su contribución a la estructura de la comunidad es mínima. Sin embargo, pueden colonizar el hábitat agrícola y por lo tanto ser calificadas como arvenses ocasionales. De la mayoría se desconoce su potencial dañino y época de invasión al hábitat agrícola en la zona de estudio; aunque existen registros del grado de nocividad de algunas como Eichhornia crassipes y Bacopa monnieri, que son consideradas como malezas perjudiciales en otros países (Holm et al., 1979).

Relaciones de la riqueza y composición de especies con el suelo

Algunos autores (Mahmood et al., 1994; Jakhar et al., 2005) han resaltado la importancia que las diferentes características edáficas ejercen en los cambios de es- 
tructura y composición florística en las comunidades de malezas que se desarrollan en suelos salinos. Con el objeto de explorar las relaciones existentes entre algunos factores fisicoquímicos del suelo y la riqueza de especies en la región de estudio se realizaron las correlaciones correspondientes sin que se vislumbrara alguna tendencia que permita establecer el efecto de ciertos rasgos edáficos sobre dicha diversidad.

Sin embargo, al explorar las preferencias que tienen las plantas por los diferentes tipos de textura y $\mathrm{pH}$, se encontró que del total de 133 especies, 17 se distribuían exclusivamente en suelos de textura franco arcillosa, 17 en los de la arcillosa, 4 en los de la franco arcilloso-arenosa, 3 en los de franco arenosa y 12 en los de la franco (Cuadro 7).

Con respecto a las tolerancias de $\mathrm{pH}$ (ver Cuadro 8), resulta claro que un buen contingente de especies (31) soportan alcalinidad elevada (más de 8.0), especialmente las 13 de la última columna que crecen en sitios con valores de $\mathrm{pH}$ de $9.0 \mathrm{o}$ más. De estas 31 plantas, 16 pertenecen a las familias Compositae (8) y Gramineae (8). Del total de las 42 especies que se encontraron con una marcada preferencia por algún tipo de $\mathrm{pH}$, no se observaron poblaciones con abundancia considerable, con excepción de Acalypha subviscida y Flaveria trinervia.

El significado de estas preferencias deberá ser evaluado en investigaciones posteriores, pero es de esperar que con la tendencia a la desecación del cuerpo de agua del Lago de Cuitzeo, las especies tolerantes a la salinidad son posibles colonizadoras de los suelos emergentes; se sugiere realizar investigaciones tendientes a conocer la biología de estas plantas, especialmente de aquellas que presentan un potencial de uso importante como los pastos (forrajeros), las comestibles, las medicinales y las que retienen el suelo, que podrían contribuir a la restauración ecológica.

\section{AGRADECIMIENTOS}

Agradecemos al Dr. Francisco Javier Espinosa la revisión y sugerencias al manuscrito, así como la traducción del resumen; al Dr. Victor W. Steinmann por la revisión y corrección de la identificación de plantas de la familia Euphorbiaceae, y a dos árbitros anónimos que mejoraron sustancialmente el trabajo. Esta investigación deriva de la tesis de licenciatura de la primera autora y forma parte del proyecto "Arvenses de la Cuenca de Cuitzeo, Michoacán", financiado por la Coordinación de la Investigación Científica de la Universidad Michoacana de San Nicolás de Hidalgo. 
Cuadro 7. Especies de plantas arvenses agrupadas por su preferencia de diferentes texturas de suelo.

\section{Franco arcillosa (17 spp.)}

Acacia farnesiana var. farnesiana

Acalypha infesta

Acalypha mexicana

Acalypha aff. subviscida

Cyclospermum leptophyllum

Anagallis arvensis

Bidens bigelovii

Cenchrus echinatus

Chloris radiata

Fleischmannia pycnocephala

Euphorbia hyssopifolia

Lepidium virginicum

Oxalis tetraphylla

Schkuhria schkuhrioides

Sida rhombifolia

Acmella alba

Tripogandra purpurascens

Franco (12 spp.)

Chloris virgata

Digitaria ternata

Eleusine indica

Euphorbia heterophylla

Heterosperma pinnatum

Melampodium microcephalum

Melampodium perfoliatum

Melampodium sericeum

Mentzelia aspera

Oxalis pes-caprae

Panicum fasciculatum

Setaria adhaerens

\section{Arcillosa (17 spp.)}

Acalypha subviscida

Commelina diffusa

Euphorbia anychioides

Eclipta prostrata

Euphorbia hirta

Hydrocotyle ranunculoides

Kallstroemia rosei

Malvella leprosa

Melampodium glabrum

Paspalum distachyon

Petunia parviflora

Plantago major

Polygonum mexicanum

Rumex pulcher spp. pulcher

Sida linearis

Stachys agraria

Tridax coronopifolia

Franco arenosa (3 spp.)

Eragrostis mexicana

Flaveria trinervia

Physalis philadelphica

Franco arcilloso-arenosa (4 spp.)

Chloris submutica

Dalea aff. versicolor

Gronovia scandens

Leptochloa fascicularis 
Cuadro 8. Especies de plantas arvenses agrupadas por sus preferencias del $\mathrm{pH}$ del suelo.

\begin{tabular}{|c|c|c|c|}
\hline pH 6.0-6.9 (4 spp.) & pH 7.0-7.9 (7 spp.) & pH 8.0-8.9 (18 spp.) & pH 9.0-9.1 (13 spp.) \\
\hline $\begin{array}{l}\text { Chloris submutica } \\
\text { Dalea aff. versicolor } \\
\text { Gronovia scandens } \\
\text { Leptochloa } \\
\text { fascicularis }\end{array}$ & $\begin{array}{l}\text { Bidens bigelovii } \\
\text { Euphorbia } \\
\text { hyssopifolia } \\
\text { Malvella leprosa } \\
\text { Melampodium } \\
\text { glabrum } \\
\text { Polygonum } \\
\text { mexicanum } \\
\text { Tridax coronopifolia } \\
\text { Sida rhombifolia }\end{array}$ & $\begin{array}{l}\text { Acalypha subviscida } \\
\text { Ambrosia psilostachya } \\
\text { Chloris radiata } \\
\text { Crotalaria pumila } \\
\text { Cyperus esculentus } \\
\text { Eclipta prostrata } \\
\text { Fleischmannia } \\
\text { pycnocephala } \\
\text { Euphorbia nutans } \\
\text { Eragrostis mexicana } \\
\text { Flaveria trinervia } \\
\text { Melampodium } \\
\text { perfoliatum } \\
\text { Oxalis pes-caprae } \\
\text { Petunia parviflora } \\
\text { Plantago major } \\
\text { Physalis philadelphica } \\
\text { Schkuhria } \\
\text { schkuhrioides } \\
\text { Setaria adhaerens } \\
\text { Solanum rostratum }\end{array}$ & $\begin{array}{l}\text { Anagallis arvensis } \\
\text { Chloris virgata } \\
\text { Dactyloctenium } \\
\text { aegyptium } \\
\text { Digitaria ternata } \\
\text { Eleusine indica } \\
\text { Euphorbia } \\
\quad \text { heterophylla } \\
\text { Heliopsis annua } \\
\text { Kallstroemia rosei } \\
\text { Lepidium virginicum } \\
\text { Melampodium } \\
\text { sericeum } \\
\text { Mentzelia aspera } \\
\text { Oxalis tetraphylla } \\
\text { Panicum fasciculatum }\end{array}$ \\
\hline
\end{tabular}

\section{LITERATURA CITADA}

Alanís, G. J. 1974. Estudio florístico-ecológico de las malezas en la región citrícola de Nuevo León, México. Publicaciones Biológicas, Universidad Autónoma de Nuevo León. 1(5): 41-64.

Altieri, A. 1987. Agroecology: The scientific basis of alternative agriculture. Westview Prees. Boulder, USA. 227 pp.

Alvarado-Díaz, J., T. Zubieta-Rojas, R. Ortega-Murillo, A. Chacón y R. Espinoza. 1984. Hipertroficación en un lago tropical somero (Lago de Cuitzeo, Michoacán, México). Revista Biológicas. Universidad Michoacana de San Nicolás de Hidalgo 1: 1-22.

Beetle, A. A., R. Guzmán M., V. Jaramillo L., M. P. Guerrero S., E. Manrique F., A. Chimal H., C. Shariff B. e I. Núñez T. 1983. Las gramíneas de México. Tomo I. Comisión Técnico-Consultiva de Coeficientes de Agostaderos, Secretaría de Agricultura y Recursos Hidráulicos. Editorial Calypso. México, D.F., México. 259 pp. 
Beetle, A. A., E. Manrique F., V. Jaramillo L., P. Guerrero S., J. Miranda S., I. Nuñez T. y A. Chimal H. 1987. Las gramíneas de México. Tomo II. Comisión Técnico-Consultiva de Coeficientes de Agostaderos. Secretaría de Agricultura y Recursos Hidráulicos. Editorial Calypso. México, D.F., México. 344 pp.

Beetle, A. A., E. Manriquez F., J. A. Miranda S., V. Jaramillo L., A. Chimal H. y A. M. Rodríguez R. 1991. Las gramíneas de México. Tomo III. Comisión TécnicoConsultiva de Coeficientes de Agostaderos. Secretaría de Agricultura y Recursos Hidráulicos. Editorial Calypso. México, D.F., México. 332 pp.

Beetle, A. A., J. A. Miranda S., V. Jaramillo L., A. M. Rodríguez R., L. Aragón M., M. A. Vergara B., A. Chimal H. y O. Domínguez S. 1995. Las gramíneas de México. Tomo IV. Comisión Técnico-Consultiva de Coeficientes de Agostaderos. Secretaría de Agricultura Ganadería y Desarrollo Rural. Editorial Calypso. México, D.F., México. 342 pp.

Bernal, M. M. y F. Guevara-Féfer. 2003. Estudio florístico de las arvenses en cultivos de maíz de temporal en los alrededores de Villa Morelos, Michoacán, México. Revista Biológicas. Universidad Michoacana de San Nicolás de Hidalgo 5: 83-93.

Chávez C., A. 1996a. El deterioro ambiental en la Cuenca del Lago de Cuitzeo. Monografía del Foro de Análisis de la Problemática Ambiental del Estado de Michoacán. Cuenca del Lago de Cuitzeo. LXVII Legislatura y Universidad Michoacana de San Nicolás de Hidalgo. Morelia, Michoacán, México. 116-117.

Chávez C., M. A. 1996b. Estudio florístico y ecológico de las plantas arvenses en cultivos de maíz de temporal en el Valle de Morelia, Michoacán, México. Tesis de licenciatura. Facultad de Biología, Universidad Michoacana de San Nicolás de Hidalgo. Morelia, Michoacán. 93 pp.

Chávez C., M. A. y F. Guevara-Féfer. 2003. Flora arvense asociada al cultivo de maíz de temporal en el Valle de Morelia, Michoacán, México. Flora del Bajío y de Regiones Adyacentes XIX. 1-22.

Corona, J. 1976. Cuitzeo (estudio antropogeográfico). La voluntad es el destino. Balsas Editores. Morelia, México. 94 pp.

Espinosa-García, F. J. 1981. Las malezas ¿una maldición? Naturaleza 5: 297-307.

Espinosa-García, F. J. y R. P. Díaz. 1996. El uso campesino de plantas arvenses como forraje en el Valle de México. Etnoecológica 3: 83-94.

Espinosa-García, F. J. y J. Sarukhán. 1997. Manual de malezas del Valle de México. Universidad Nacional Autónoma de México - Fondo de Cultura Económica. México, D.F., México. 407 pp.

García, E. 1988. Modificaciones al sistema de clasificación climática de Köppen. 4a. ed. Instituto de Geografía, Universidad Nacional Autónoma de México. México, D.F., México. 246 pp.

Guevara-Féfer, F. 1995. Vegetación del Valle de Morelia, Michoacán. Revista Biológicas. Universidad Michoacana de San Nicolás de Hidalgo 3: 20-54.

Guevara-Féfer, F., R. Sánchez B. y A. Espinoza de los M. 1984. Informe Técnico. Proyecto: Estudio florístico ecológico de las comunidades de arvenses en cultivos de maíz en el Valle de Morelia. Convenio-Escuela de Biología Universidad Michoacana de San Nicolás de Hidalgo-Consejo Nacional de Ciencia y Tecnología. 100 pp.

Holm, L., J. V. Pancho, J. P. Herberger y D. L. Plucknett. 1979. A geographical atlas of world weeds. John Wiley \& Sons. New York, USA. 391 pp. 
Jakhar, G. S., S. A. Abro, A. Q. Maher y R. Qureshi. 2005. Weed communities of wheat crop under diverse edaphogropghy of district Khaipur, Pakistan. Pakistan J. Bot. 37: 709-714.

Kovach, W. L. 2005. A MultiVariate Statistical Package for Windows, (MVSP) version 3.1. Kovach Computing Services. Pentraeth, Wales, U.K.

Krebs, J. C. 1989. Ecological methodology. University of Columbia. Harper \& Row Publishers. New York, USA. 654 pp.

Mahmood, K., K. A. Malik, M. A. K. Lodhi y K. H. Sheikh. 1994. Soil-plant relationships in saline wastelands: vegetation, soils, and successional changes, during biological amelioration. Environ. Conserv. 21: 236-241.

Marzocca, A. 1976. Manual de malezas. 3a. ed. Hemisferio Sur. Buenos Aires, Argentina. 564 pp.

McVaugh, R. 1983. Gramineae. Flora Novo-Galiciana 14: 1-436.

McVaugh, R. 1984. Compositae. Flora Novo-Galiciana 12: 1-1157.

Mueller-Dombois, D. y H. Ellenberg. 1974. Aims and methods of vegetation ecology. John Wiley \& Sons. New York, USA. 547 pp.

Rojas, J. y A. Novelo. 1995. Flora y vegetación acuática del Lago Cuitzeo, Michoacán, México. Acta Bot. Mex. 31: 1-17.

Rzedowski, J. 1991. Diversidad y orígenes de la flora fanerogámica de México. Acta Bot. Mex. 14: 3-21.

Rzedowski, J. y G. C. Rzedowski. 1979. Flora fanerogámica del Valle de México. Vol I. CECSA. México, D.F., México. 402 pp.

Rzedowski, J. y G. C. Rzedowski. 1985. Flora fanerogámica del Valle de México. Vol II. Dicotyledoneae. Escuela Nacional de Ciencias Bilógicas, Instituto Politécnico Nacional e Instituto de Ecología. México, D.F., México. 674 pp.

Rzedowski, J. y G. C. Rzedowski. 1990. Flora fanerogámica del Valle de México. Vol III. Monocotyledoneae. Centro Regional del Bajío, Instituto de Ecología, A.C., Pátzcuaro, Michoacán, México. 674 pp.

Sen, D. N. 1981. Ecological approach to Indian weeds. Geographic Market and Business Intelligence. Jodhpur, India. $301 \mathrm{pp}$.

Torres B., A. 1991. Interacciones y comportamiento de las plantas arvenses en mono y policultivos de maíz. Tesis profesional. Facultad de Ciencias, Universidad Nacional Autónoma de México. México, D.F., México. 63 pp.

Vieyra-Odilon, L. y H. Vibrans. 2001. Weeds as crops: The value of maize field weeds in the valley of Toluca, México. Econ. Bot. 55: 426-446.

Villaseñor R., J. L. y F. J. Espinosa-García. 1998. Catálogo de malezas de México. Universidad Nacional Autónoma de México-Consejo Nacional Consultivo Fitosanitario - Fondo de Cultura Económica. México, D.F., México. 449 pp.

Villaseñor R., J. L. y F. J. Espinosa-García. 2004. The alien flowering plants of Mexico. Divers. Distrib. 10: 113-123. 


\section{APÉNDICE}

Lista de las especies arvenses en cultivos de maíz de temporal en suelos salinos de la ribera del Lago de Cuitzeo, Michoacán, México. F/V: Forma de vida, A: Anual, P: Perenne, A/P: Anual-Perenne, A/B: Anual-Bianual; (+) Especies leñosas; ( $\bullet$ Especies introducidas; $(*)$ Nuevos registros.

\begin{tabular}{|c|c|c|}
\hline Nombre científico & Nombre común & $\mathrm{F} / \mathrm{V}$ \\
\hline \multicolumn{3}{|l|}{ ACANTHACEAE } \\
\hline Dicliptera penduncularis Nees & - & A \\
\hline \multicolumn{3}{|l|}{ AIZOACEAE } \\
\hline Trianthema portulacastrum $\mathrm{L}$. & Verdolaga de burro & A \\
\hline \multicolumn{3}{|l|}{ AMARANTHACEAE } \\
\hline Amaranthus hybridus L. & Quelite & A \\
\hline Gomphrena serrata L. & Amor seco & A-P \\
\hline \multicolumn{3}{|l|}{ BORAGINACEAE } \\
\hline Heliotropium curassavicum $\mathrm{L}$. & Cola de mico & A-P \\
\hline \multicolumn{3}{|l|}{ CHENOPODIACEAE } \\
\hline •Chenopodium murale L. & Leche de perico & A \\
\hline \multicolumn{3}{|l|}{ COMMELINACEAE } \\
\hline Commelina diffusa Burm. f. & Siempre viva & A-P \\
\hline Tripogandra purpurascens (S. Schauer) Handlos & Pico de pollo & A \\
\hline \multicolumn{3}{|l|}{ COMPOSITAE (ASTERACEAE) } \\
\hline Acmella alba (L’Hér.) R.K. Jansen & Chile burro & A-P \\
\hline Ambrosia psilostachya DC. & Amargosa, altamisa & $\mathrm{P}$ \\
\hline Bidens aurea (Aiton) Sherff & Té de milpa & A-P \\
\hline *Bidens bigelovii A. Gray & - & A \\
\hline Bidens odorata Cav. & Aceitilla & A \\
\hline Dyssodia papposa (Vent.) Hitchc. & Flor de muerto & A \\
\hline *Dyssodia tagetiflora Lag. & - & A \\
\hline Eclipta prostrata $(\mathrm{L}.) \mathrm{L}$. & Epazotillo & $\mathrm{P}$ \\
\hline Flaveria trinervia (Spreng.) C. Mohr & Rompe calzón & A \\
\hline *Fleischmannia pycnocephala (Less.) R.M. King \& H. Rob. & - & $\mathrm{P}$ \\
\hline Florestina pedata (Cav.) Cass. & Jarilla & A \\
\hline Galinsoga parviflora Cav. & $\begin{array}{l}\text { Granjenillo, rosa } \\
\text { amarilla }\end{array}$ & A \\
\hline Galinsoga quadriradiata Ruiz \& Pav. & Estrellita & A \\
\hline
\end{tabular}


Apéndice. Continuación.

\begin{tabular}{|c|c|c|}
\hline Nombre científico & Nombre común & $\mathrm{F} / \mathrm{V}$ \\
\hline Heliopsis annua Hemsl. & - & A \\
\hline Heterosperma pinnatum Cav. & Jarilla & A \\
\hline *Melampodium glabrum S. Watson & - & A \\
\hline *Melampodium microcephalum Less. & - & A \\
\hline Melampodium perfoliatum (Cav.) Kunth & Andancillo & A \\
\hline Melampodium sericeum Lag. & - & A \\
\hline Parthenium bipinnatifidum (Ortega) Rollins & Confitillo & A \\
\hline Sanvitalia procumbens Lam. & Ojo de pollo & A \\
\hline Schkuhria schkuhrioides Thell. & Cincollagas & A \\
\hline Simsia amplexicaulis (Cav.) Pers. & Andán colorado & A \\
\hline Sonchus oleraceus L. & Lechuguilla & A \\
\hline *Tagetes lunulata Ortega & Cinco llagas & A \\
\hline Tithonia tubiformis (Jacq.) Cass. & Andán & A \\
\hline Tridax coronopifolia (Kunth) Hemsl. & Motitas & A-P \\
\hline Xanthium strumarium L. & Chayotillo, abrojo & A \\
\hline *Zinnia peruviana (L.) L. & Mal de ojo & A \\
\hline \multicolumn{3}{|l|}{ CONVOLVULACEAE } \\
\hline Ipomoea dumetorum Willd. ex Roem. \& Schult. & - & A \\
\hline Ipomoea purpurea (L.) Roth & Quebraplato & A \\
\hline \multicolumn{3}{|l|}{ CRUCIFERAE (BRASSICACEAE) } \\
\hline Lepidium virginicum $\mathrm{L}$. & Lentejilla & $\mathrm{A}-\mathrm{B}$ \\
\hline \multicolumn{3}{|l|}{ CUCURBITACEAE } \\
\hline Sicyos deppei G. Don & Chayotillo & A \\
\hline \multicolumn{3}{|l|}{ CYPERACEAE } \\
\hline •yperus esculentus L. & Zacate, tulillo & $\mathrm{P}$ \\
\hline $\begin{array}{l}\text { *Schoenoplectus americanus (Pers.) Volkart ex Schinz \& R. } \\
\text { Keller }\end{array}$ & Tule & $\mathrm{P}$ \\
\hline \multicolumn{3}{|l|}{ EUPHORBIACEAE } \\
\hline Acalypha infesta Poepp. & - & A \\
\hline Acalypha mexicana Mull. Arg. & Hierba del cáncer & A \\
\hline Acalypha subviscida $\mathrm{S}$. Watson & - & A \\
\hline Acalypha aff. subviscida S. Watson & - & A \\
\hline *Euphorbia anychioides Boiss. & - & $\mathrm{P}$ \\
\hline *Euphorbia glyptosperma Engelm. & - & A \\
\hline
\end{tabular}


Apéndice. Continuación.

\begin{tabular}{lcc}
\hline Nombre científico & Nombre común & F/V \\
\hline Euphorbia graminea Jacq. & - & A \\
Euphorbia heterophylla L. & Lechosa & A \\
$*$ Euphorbia hirta L. & - & A \\
*Euphorbia hyssopifolia L. & Hierba de la & A \\
& golondrina & \\
$*$ Euphorbia mendezii Boiss. & Alfombrilla & A \\
Euphorbia nocens (L.C. Wheeler) V.W. Steinm. & Golondrina & A \\
$*$ Euphorbia nutans Lag. & - & A-P \\
Ricinus communis L. & Higuerilla & A-P
\end{tabular}

GRAMINEAE (POACEAE)

Bromus carinatus Hook. \& Arn.

Cebadillo, avena $\quad \mathrm{P}$

loca

Cenchrus echinatus L.

A

*Chloris radiata (L.) Sw.

*Chloris submutica Kunth

-Chloris virgata Sw.

Grama

A

$\checkmark$ Cynodon dactylon (L.) Pers.

Pelo de conejo

-Dactyloctenium aegyptium (L.) Willd.

- Digitaria ciliaris (Retz.) Koeler

-Digitaria ternata (Hochst. ex A. Rich.) Stapf

$\checkmark$ Echinochloa colonum (L.) Link

Pata de gallo

A

$\checkmark$ Echinochloa crus-galli (L.) P. Beauv.

Grama

A

-Eleusine indica (L.) Gaertn.

Camalote

Eragrostis pectinacea (Michx.) Nees

Camalote

A

Eragrostis mexicana (Hornem.) Link

Grama cabezona

*Eriochloa acuminata (J. Presl) Kunth

\section{Grama}

Leersia hexandra Sw.

Leptochloa fascicularis (Lam.) A. Gray

Grama, zacate

Oplismenus burmannii (Retz.) P. Beauv.

Grama, zacate

Panicum decolorans Kunth

Zacate granillo

*Panicum fasciculatum Sw.

Grama, zacate

Panicum hirticaule J. Presl

Grama, zacate

Paspalum distichum L.

Grama, zacate

A

$\checkmark$ Paspalum dystachion Poit. ex Trin.

Liendrilla, sabana 
Apéndice. Continuación.

\begin{tabular}{|c|c|c|}
\hline Nombre científico & Nombre común & $\mathrm{F} / \mathrm{V}$ \\
\hline Paspalum lividum Trin. ex Schltdl. & Grama, zacate & $\mathrm{P}$ \\
\hline • Setaria adhaerens (Forssk.) Chiov. & Tripa de gallina & A \\
\hline Setaria grisebachii E. Fourn. & Grama & A-B \\
\hline Setaria parviflora (Poir.) Kerguélen & Zacate sedoso & $\mathrm{P}$ \\
\hline Urochloa plantaginea (Link) R.D. Webster & Zacate panizo & A \\
\hline Zea mays subsp. mexicana (Schrad.) Iltis & Condax & A \\
\hline \multicolumn{3}{|l|}{ LABIATAE (LAMIACEAE) } \\
\hline Salvia tiliifolia Vahl & Tronadora & A \\
\hline Stachys agraria Schltdl. \& Cham. & Toronjil & A-P \\
\hline \multicolumn{3}{|l|}{ LEGUMINOSAE (FABACEAE) } \\
\hline +Acacia farnesiana (L.) Willd. var. farnesiana & Huizache & $\mathrm{P}$ \\
\hline Crotalaria pumila Ortega & Huajilla & A \\
\hline Dalea aff. versicolor Zucc. & - & A \\
\hline -Dalea leporina (Aiton) Bullock & - & A \\
\hline -Medicago lupulina L. & - & A \\
\hline $\begin{array}{l}\text { +Prosopis laevigata (Humb. \& Bonpl. ex Willd.) M. C. } \\
\text { Johnst. }\end{array}$ & Mezquite & $\mathrm{P}$ \\
\hline \multicolumn{3}{|l|}{ LOASACEAE } \\
\hline *Gronovia scandens L. & Pegarropa & $\mathrm{P}$ \\
\hline Mentzelia aspera L. & - & A \\
\hline
\end{tabular}

MALVACEAE

Anoda cristata (L.) Schltdl.

Estrella, malva A

* Malvella leprosa (Ortega) Krapov.

- $\quad P$

+Sida linearis Cav.

Malvavisco $\quad \mathrm{P}$

Sida rhombifolia L.

NYCTAGINACEAE

+Mirabilis nyctaginea (Michx.) MacMill.

Maravillita A

ONAGRACEAE

Lopezia racemosa Cav.

*Oenothera kunthiana (Spach) Munz

Alfilerillo, lentejilla A-P

Oenothera pubescens Willd. ex Spreng.

OXALIDACEAE

Oxalis corniculata L.

Agritos

$\mathrm{P}$

* Oxalis pes-caprae L.

Xocoyole

$\mathrm{P}$ 
Apéndice. Continuación.

\begin{tabular}{lcc}
\hline Nombre científico & Nombre común & $\mathrm{F} / \mathrm{V}$ \\
\hline Oxalis tetraphylla Cav. & - & $\mathrm{P}$
\end{tabular}

PAPAVERACEAE

Argemone ochroleuca Sweet

Lechuga, chicalote A-P

PASSIFLORACEAE

*Passiflora exsudans Zucc.

Pasionaria

$\mathrm{P}$

PLANTAGINACEAE

-Plantago major L.

Juan primero

A-P

PLUMBAGINACEAE

*Plumbago pulchella Boiss.

POLYGONACEAE

•Polygonum lapathifolium L.

Hierba del negro $\quad \mathrm{P}$

Polygonum mexicanum Small

Chilillo

A-P

Rumex flexicaulis Rech. f.

Lengua de vaca

A

cimarrona

A-B

^Rumex pulcher L. ssp. pulcher

PONTEDERIACEAE

-Eichhornia crassipes (Mart.) Solms

Lirio

$\mathrm{P}$

PORTULACACEAE

Portulaca oleracea L.

Verdolaga silvestre

A

PRIMULACEAE

-Anagallis arvensis L.

Hierba del pájaro

A

RUBIACEAE

Spermacoce confusa Rendle

Chilquilite

$\mathrm{P}$

SCROPHULARIACEAE

*Bacopa monnieri (L.) Wettst.

Castilleja arvensis Schltdl. \& Cham.

Cresta de gallo

SOLANACEAE

+Lycium carolinianum Walter

Tomate de burro

- Nicandra physalodes (L.) Gaertn.

Petunia parviflora Juss.

A

Physalis philadelphica Lam.

Tomate

A

Physalis sulphurea (Fernald) Waterf.

Tomatillo

A

Solanum americanum Mill.

Hierba mora

A

Solanum rostratum Dunal

Mala mujer

A-P

A 
Apéndice. Continuación.

\begin{tabular}{lcc}
\hline Nombre científico & Nombre común & F/V \\
\hline UMBELLIFERAE (APIACEAE) & & \\
Cyclospermum leptophyllum (Pers.) Sprague & Cilantro cimarrón & A-P \\
Hydrocotyle ranunculoides L. f. & Ombligo de Venus & P \\
VERBENACEAE & & \\
Bouchea prismatica (L.) Kuntze & - & $\mathrm{A}$ \\
Glandularia bipinnatifida (Nutt.) Nutt. & - & $\mathrm{P}$ \\
ZYGOPHYLLACEAE & - & \\
Kallstroemia rosei Rydb. & - & $\mathrm{A}$ \\
\hline
\end{tabular}


